\title{
Phase-Dependent Interactions in Visual Cortex to Combinations of First- and Second-Order Stimuli
}

\author{
Claire V. Hutchinson, ${ }^{1}$ (T) Timothy Ledgeway, ${ }^{2}$ and Curtis L. Baker, $\mathrm{Jr}^{3}$ \\ ${ }^{1}$ College of Medicine, Biological Sciences and Psychology, University of Leicester, Leicester LE1 9HN, United Kingdom, ${ }^{2}$ School of Psychology, University of \\ Nottingham, Nottingham NG7 2RD, United Kingdom, and ${ }^{3}$ Vision Research Unit, McGill University Health Centre Research Institute, Department of \\ Ophthalmology, McGill University, Montreal, Quebec, Canada H3A 1A1
}

A fundamental task of the visual system is to extract figure- ground boundaries between objects, which are often defined, not only by differences in luminance, but also by "second-order" contrast or texture differences. Responses of cortical neurons to both first- and second-order patterns have been studied extensively, but only for responses to either type of stimulus in isolation. Here, we examined responses of visual cortex neurons to the spatial relationship between superimposed periodic luminance modulation (LM) and contrast modulation (CM) stimuli, the contrasts of which were adjusted to give equated responses when presented alone. Extracellular single-unit recordings were made in area 18 of the cat, the neurons of which show responses to CM and LM stimuli very similar to those in primate area V2 (Li et al., 2014). Most neurons showed a significant dependence on the relative phase of the combined LM and CM patterns, with a clear overall optimal response when they were approximately phase aligned. The degree of this phase preference, and the contributions of suppressive and/or facilitatory interactions, varied considerably from one neuron to another. Such phase-dependent and phaseinvariant responses were evident in both simple- and complex-type cells. These results place important constraints on any future model of the underlying neural circuitry for second-order responses. The diversity in the degree of phase dependence between LM and CM stimuli that we observed could help to disambiguate different kinds of boundaries in natural scenes.

Key words: contrast modulation; first-order; form-cue invariance; second-order; spatial phase; visual cortex

\section{Significance Statement}

Many visual cortex neurons exhibit orientation-selective responses to boundaries defined by differences either in luminance or in texture contrast. Previous studies have examined responses to either type of boundary in isolation, but here we measured systematically responses of cortical neurons to the spatial relationship between superimposed periodic luminance-modulated (LM) and contrast-modulated (CM) stimuli with contrasts adjusted to give equated responses. We demonstrate that neuronal responses to these compound stimuli are highly dependent on the relative phase between the LM and CM components. Diversity in the degree of such phase dependence could help to disambiguate different kinds of boundaries in natural scenes, for example, those arising from surface reflectance changes or from illumination gradients such as shading or shadows.

\section{Introduction}

Natural scenes contain a multiplicity of complex features that provide important information concerning object position, surface structure, boundaries and contours, spatial scale, motion, and rela-

Received April 25, 2016; revised 0ct. 7, 2016; accepted 0ct. 11, 2016.

Author contributions: C.V.H., T.L., and C.L.B. designed research; C.V.H., T.L., and C.L.B. performed research; C.V.H., T.L., and C.L.B. analyzed data; C.V.H., T.L., and C.L.B. wrote the paper.

This work was supported by the Canadian Institutes of Health Research (Operating Grants MOP-119498 and MOP-9685 to C.B.). C.V.H was supported in part by a Human Frontier Science Program Organization Short-Term

Fellowship. We thank Lynda Domazet, Guangxing Li, and Vargha Talebi for assistance with some of the experiments. The authors declare no competing financial interests.

Correspondence should be addressed to Curtis L. Baker, Jr., McGill Vision Research, MUHC Research Institute, 1650 Cedar Ave, Room L11.112, Montreal, Quebec, Canada, H3G 1A4. E-mail: curtis.baker@McGill.ca.

DOI:10.1523/JNEUROSCI.1350-16.2016

Copyright $\odot 2016$ the authors $\quad 0270-6474 / 16 / 3612328-10 \$ 15.00 / 0$ tive distance. The visual system uses these cues to detect and identify objects in a scene by segregating them from their background. An object may be delineated from its background by intensive "firstorder” properties - for example, variations in luminance or color within different regions of the image- or by more complex "second-order" attributes, in which areas are differentiated by cues such as contrast, texture, relative motion, and binocular disparity. In natural images, there is a highly structured spatial relationship between occurrences of first- and second-order information (Schofield, 2000; Johnson and Baker, 2004). Human psychophysical studies show that combined first- and second-order cues improve texture segmentation (Smith and Scott-Samuel, 1998; Johnson et al., 2007) and could potentially be used to help resolve ambiguities in first-order information, for example, to distinguish surface reflec- 
A
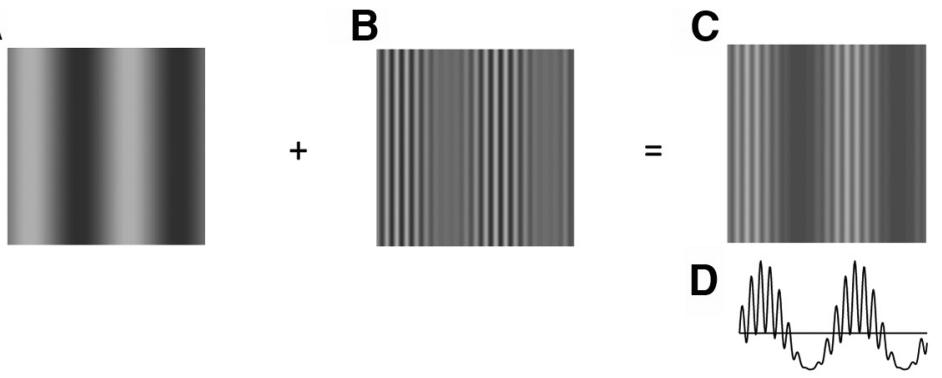

$E$

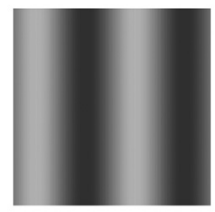

$\mathbf{F}$

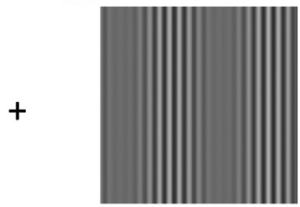

G

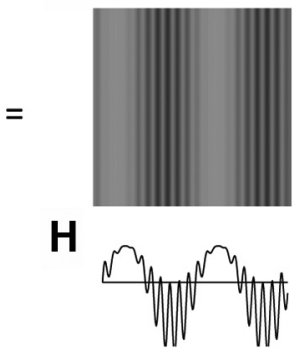

Figure 1. Examples of stimulus composition for main experiment, in which one $L M$ phase is combined with two different $C M$ phases. $\boldsymbol{A}-\boldsymbol{C}, \mathrm{LM}(\boldsymbol{A})$ added to $C M$ of the same spatial phase $(\boldsymbol{B})$ produced an in-phase $\left(0^{\circ}\right.$ offset) composite stimulus $(\boldsymbol{C})$. Note that only the contrast variations about the mean background were added, as detailed in Equation 5. Luminance and contrast modulations (LM and $\mathrm{CM}$ ) were taken to be in-phase when high- and low-luminance and high- and low-contrast bars of the grating and envelope, respectively, were phase aligned. $\boldsymbol{D}, 1 \mathrm{D}$ luminance profile corresponding to stimulus image in $\boldsymbol{C}$. $\boldsymbol{E}-\boldsymbol{H}$, Same as $\boldsymbol{A}-\boldsymbol{D}$ but the component patterns were summed in antiphase $\left(180^{\circ}\right.$ relative phase offset), producing a composite stimulus $G$ in which the high-and low-luminance bars of the grating were centered on the low- and high-contrast bars of the envelope, respectively. See text for further details.

tance versus illumination effects (Schofield et al., 2006, 2010; Sun and Schofield, 2011).

Neurons responsive to both first- and second-order stimuli are evident in many visual cortical areas (V1, V2, and V5/MT) of the monkey (Albright, 1992; Chaudhuri and Albright, 1997; but see ElShamayleh and Movshon, 2011; Li et al., 2014) and areas 17 and 18 of the cat (Zhou and Baker, 1994; Tanaka and Ohzawa, 2006; Rosenberg and Issa, 2011). Many of these demonstrate form-cue invariance to first- and second-order motion patterns in that they respond to either kind of stimulus with consistent direction selectivity and preferred orientation (Albright, 1992; Geesaman and Anderson, 1996; Mareschal and Baker, 1999; Li et al., 2014). Human fMRI also reveals orientation- or direction-selective responses to first- and second-order stimuli in many extrastriate cortical areas and in primary visual cortex (Nishida et al., 2003; Seiffert et al., 2003; Larsson et al., 2006; Hallum et al., 2011).

In natural images, first- and second-order information often occurs at coincident locations (Johnson and Baker, 2004) such as at occlusion boundaries. Therefore, it is important to understand how these two types of information are combined in visual cortex. However, previous neurophysiological studies have only examined neuronal responses to first- or second-order stimuli in isolation. Here, we measured systematically responses of cortical neurons to the spatial relationship between superimposed periodic luminance-modulated (LM) and second-order contrastmodulated (CM) stimuli with contrasts adjusted to give equated responses. These recordings were done in area 18 of the cat, in which neurons show CM and LM responses largely similar to those in macaque area V2 (Li et al., 2014). We found that many of the neurons exhibited responses to compound stimuli that were highly dependent on the relative phase between the LM and CM components, with differing degrees of suppressive and/or facilitatory interactions in different neurons. Such phase-dependent

and phase-invariant responses are evident in both simple- and complex-type cells.

\section{Materials and Methods}

Animal preparation and maintenance. Initial anesthesia of adult cats of either sex was induced by isoflourane/oxygen (3-5\%) inhalation, followed by intravenous cannulation and bolus intravenous delivery of thiopentone sodium $(8 \mathrm{mg} / \mathrm{kg})$ or propofol $(5 \mathrm{mg} / \mathrm{kg})$, atropine sulfate $(0.05 \mathrm{mg} / \mathrm{kg})$, and dexamethasone $(0.2 \mathrm{mg} / \mathrm{kg})$. The corneas were protected during surgery with topical carboxymethylcellulose (1\%). Surgical anesthesia was maintained with supplemental doses of thiopentone as required or with propofol $(6 \mathrm{mg} / \mathrm{kg} / \mathrm{h})$ and all surgical wounds were infused with bupivacaine $(0.25 \%)$. A secure airway was established by tracheal cannulation or intubation. A craniotomy (H-C A3/L4) provided access to cortical area 18 (Tusa et al., 1979) using glass-coated platinum-iridium or parylene-coated tungsten microelectrodes (Frederick Haer). The cortical surface was protected with $2 \%$ agarose (SigmaAldrich, Type 1-A) and petroleum jelly.

After completion of surgery, animals were paralyzed with an intravenous bolus injection of gallamine triethodide $(10 \mathrm{mg} / \mathrm{kg})$, followed by infusion $(10 \mathrm{mg} / \mathrm{kg} / \mathrm{h})$. Anesthesia was maintained with sodium pentobarbital (1.0 $\mathrm{mg} / \mathrm{kg} / \mathrm{h}$ ) in earlier experiments or with fentenyl $(9 \mu \mathrm{g} / \mathrm{kg}$ bolus, then $26 \mu \mathrm{g} / \mathrm{kg} / \mathrm{h})$ and propofol $(5 \mathrm{mg} / \mathrm{kg} / \mathrm{h})$ in later experiments, supplemented with oxygen/nitrous oxide (70:30) and dextrose-saline (2 $\mathrm{ml} / \mathrm{h}$ ). Expired $\mathrm{CO}_{2}$, blood $\mathrm{O}_{2}$, heart rate, electroencephalogram, and temperature were monitored throughout the experiment and maintained at appropriate levels. Corneal protection was provided by neutral contact lenses and emmetropia at a distance of $57 \mathrm{~cm}$ was provided by spectacle lenses selected with slit retinoscopy and artificial pupils (2.5 $\mathrm{mm}$ ). All animal procedures were approved by the McGill University Animal Care Committee and are in accordance with the guidelines set out by the Canadian Council on Animal Care.

Visual stimuli. Visual stimuli were produced on a Macintosh computer (MacPro 4.1, MacOS 10.6.8, 2.66 Ghz/4 core, 6 Gb, NVIDIA GeForce GT120) using custom software written in Matlab (The Mathworks) with the Psychophysics Toolbox (Brainard, 1997; Pelli, 1997; Kleiner et al., 2007). Stimulus patterns were displayed on a CRT monitor (NEC FP1350, 20", $640 \times 480$ pixels, $75 \mathrm{~Hz}, 36 \mathrm{~cd} / \mathrm{m}^{2}$, bit depth 8) placed at a viewing distance of $57 \mathrm{~cm}$. The monitor's gamma nonlinearity was measured with a photometer (United Detector Technology) and corrected with an inverse lookup table.

Three types of stimulus patterns were used: first-order luminancemodulated (LM) gratings, second-order contrast-modulated (CM) envelopes, and a compound of the two $(\mathrm{LM}+\mathrm{CM})$. In each case, these were zero-balanced patterns of contrast against a mean luminance background, $L_{0}$.

Luminance gratings were spatially $1 \mathrm{D}$ sinusoidal modulations (Fig. $1 A, E)$ as follows:

$$
L(x, y, t)=L_{0}\left\{1+C_{L} \sin \left[2 \pi\left(\omega_{\mathrm{s}}(x \cos \theta+y \sin \theta)-\omega_{\mathrm{t}} t\right)\right]\right\}
$$

where $C_{\mathrm{L}}$ is the Michelson contrast of luminance modulation, $\omega_{\mathrm{s}}$ is spatial frequency, $\theta$ is orientation, and $\omega_{\mathrm{t}}$ is temporal frequency. The secondorder stimuli ("contrast envelopes"; Fig. $1 B, F$ ) were spatially 1D sinusoidal modulations of the contrast of a high-spatial frequency carrier grating:

$$
L(x, y, t)=L_{0}\{1+\operatorname{Carr}(x, y)[1+\operatorname{Env}(x, y, t)] / 2\}
$$


The carrier grating was a high spatial frequency, stationary sine wave grating:

$$
\operatorname{Carr}(x, y)=C_{\mathrm{c}} \sin \left[2 \pi \omega_{\mathrm{c}}\left(x \cos \theta_{\mathrm{c}}+y \sin \theta_{\mathrm{c}}\right)\right]
$$

where $C_{c}$ is the carrier contrast, $\omega_{c}$ is carrier spatial frequency, and $\theta_{c}$ is carrier orientation. The carrier was multiplied by an envelope pattern consisting of a low spatial frequency, drifting sine wave grating:

$$
\operatorname{Env}(x, y, t)=C_{\mathrm{E}} \sin \left[2 \pi\left(\omega_{\mathrm{s}}(x \cos \theta+y \sin \theta)-\omega_{\mathrm{t}} t\right)+\phi\right],
$$

where $C_{\mathrm{E}}$ is the envelope contrast, $\omega_{\mathrm{s}}$ and $\omega_{\mathrm{t}}$ are the envelope spatial and temporal frequency, respectively, and $\theta$ is the envelope orientation. The compound stimuli were superpositions of the LM and CM patterns:

$$
\begin{aligned}
L(x, y, t)=L_{0}\{\{1 & +\operatorname{Carr}(x, y)[1+\operatorname{Env}(x, y, t)] / 2\} \\
& \left.+\left\{C_{L} \sin \left[2 \pi\left(\omega_{s}(x \cos \theta+y \sin \theta)-\omega_{t} t\right)\right]\right\}\right\}
\end{aligned}
$$

Note that these three stimuli have identical envelope orientation $(\theta)$ and spatial and temporal frequencies $\left(\omega_{\mathrm{s}}, \omega_{\mathrm{t}}\right)$, but can have varying values of relative spatial phase $(\phi)$. Examples of single frames and 1D profiles are shown in Figure 1, C and $D$, and Figure 1, G and $H\left(\phi=0^{\circ}\right.$ and $\phi=180^{\circ}$, respectively). LM and CM stimuli were considered to be "in-phase" $\left(0^{\circ}\right)$ when the high and low luminance bars of the grating were centered on the high- and low-contrast bars of the envelope and "antiphase" $\left(180^{\circ}\right)$ in the opposite case; this definition was determined a priori.

Stimulus patterns were presented within a cosine-tapered circular aperture, against a uniform background at the mean luminance of the pattern. The same mean luminance was also maintained during intervals between stimuli, and was presented as blank conditions for measurement of spontaneous activity.

Electrophysiology. The microelectrode was advanced with a steppingmotor microdrive (M. Walsh Electronics). Single units were isolated with a window discriminator (Frederick Haer) and isolation was monitored on a delay-triggered oscilloscope. Manually controlled bar-shaped stimuli were used to map the receptive field approximately and determine ocular dominance. The display screen was centered on the receptive field and subsequent stimuli were delivered only to the neuron's dominant eye. Spike times were recorded with $0.1 \mathrm{~ms}$ resolution (ITC-18; Instrutech) and their temporal registration with the stimulus was established with reference to an optical sensor (T2L12S; TAOS) placed over a corner of the display containing stimulus timing information. Within an experimental run, different stimulus conditions were presented for 0.5 $1.0 \mathrm{~s}$ in randomly interleaved order $(0.5 \mathrm{~s}$ for LM gratings, $1.0 \mathrm{~s}$ for CM or $\mathrm{LM}+\mathrm{CM}$ stimuli), with 5-20 repetitions of each stimulus. Poststimulus time histograms and plots of average spike frequency as functions of varied stimulus parameters were displayed online. Spike times and stimulus information were recorded to hard disk files for subsequent detailed analysis.

Each neuron was characterized quantitatively with conventional tuning-curve measurements using first-order grating patterns to establish its optimal orientation, spatial/temporal frequency, simple/ complex classification, and location and size of its receptive field. Each neuron was assessed for responsiveness to second-order stimuli using procedures similar to those used previously (Mareschal and Baker, 1999; Tanaka and Ohzawa, 2006). Contrast envelope stimuli were presented using envelope parameters (orientation, spatial/temporal frequency) that were optimal for first-order stimuli and a series of relatively high carrier spatial frequencies were tested (typically $\sim 0.5-3.0 \mathrm{cpd}$ ). A neuron was considered envelope responsive if the data exhibited a band-pass-tuned response to the spatial frequency of the carrier, which was clearly distinct from its response to luminance gratings, such that the contrast envelope response clearly could not be mediated by the same mechanism underlying the response to firstorder gratings. Then, using this optimal carrier spatial frequency, the response to a series of carrier orientations was tested systematically to further optimize the response. All subsequent tests used these individually optimized parameters for contrast envelopes, and first-order luminance gratings were used with parameters matched to those of the second-order envelopes.

After these preliminary measurements, subsequent experiments were performed on envelope-responsive neurons. Contrast response functions (Ledgeway et al., 2005) were measured for both first-order (luminance grating) and second-order (contrast envelope) stimuli using identical values of envelope orientation and spatial/temporal frequency. From these data, contrast values for the two stimuli were selected that would produce approximately equated responses. Because neurons are typically more responsive to LM than to CM patterns, a high CM envelope contrast (typically 100\%) was chosen and the spike frequency was matched with an equivalent LM contrast. Unless otherwise noted, these values were used for the compound $(\mathrm{LM}+\mathrm{CM})$ stimuli that were presented at a series of values of relative spatial phase.

Quantitative measurements for this study were obtained from 76 neurons in nine animals. Note that this work was performed in conjunction with other studies on the same animals being conducted concurrently. Of these neurons, 28 were significantly envelope responsive and their isolation was maintained sufficiently long $(\sim 2 \mathrm{~h})$ to obtain all of the preliminary measurements and the contrast response and phase interaction datasets to qualify for inclusion in the study.

Data analysis. Spike times were collected into poststimulus time histograms (bin width $10 \mathrm{~ms}$ ) and plots of time-averaged spike frequency as functions of varied parameters were constructed. Neurons were classified as simple or complex type based on the ratio of response at the first harmonic of stimulus temporal frequency to the average firing rate (Skottun et al., 1991). Optimal parameters for descriptive mathematical functions (see below) were estimated using curve-fitting functionality of Kaleidagraph (Synergy Software) or Matlab (The Mathworks).

\section{Results}

\section{Contrast response functions}

Neurons were markedly less responsive to CM than to LM stimuli, consistent with previous studies (Ledgeway et al., 2005). To maximize the opportunity to detect interactions between the two stimuli and to ensure that the response would not be dominated by the LM stimulus, we amplitude-equated ('matched') the two stimulus types in terms of each neuron's responsiveness. This was achieved by measuring contrast response functions (CRFs) for each stimulus type using optimized stimulus parameters as outlined above. Note that, for each neuron, the orientation, spatial frequency, temporal frequency, and direction of motion of the modulation waveforms were identical for LM and CM and, in the case of CM, the optimal carrier was also used. Based on these measurements, we selected values of grating and envelope contrast that elicited an approximately equivalent response (Fig. $2 A, B$, green dashed lines). A CM carrier contrast of $70 \%$ was used throughout to ensure that the sum of carrier contrast for CM and luminance contrast for LM would be physically realizable; that is, not exceeding $100 \%$.

\section{Phase-dependent responses}

LM and CM stimuli were superimposed at their responsematched amplitudes and responses (average spikes/s) were recorded as a function of their relative spatial phase offset. In the example of a complex-type cell shown in Figure $2 C$, the response was markedly dependent on the relative spatial phase difference between LM and CM stimuli, with a peak response at a relative spatial phase somewhat greater than zero (close to phase alignment; Fig. 1C). As the spatial phase offset between the two stimuli increased, responses became less vigorous, producing the weakest responses when LM and CM stimuli were close to antiphase $\left(180^{\circ}\right.$; Fig. $\left.1 G\right)$. 


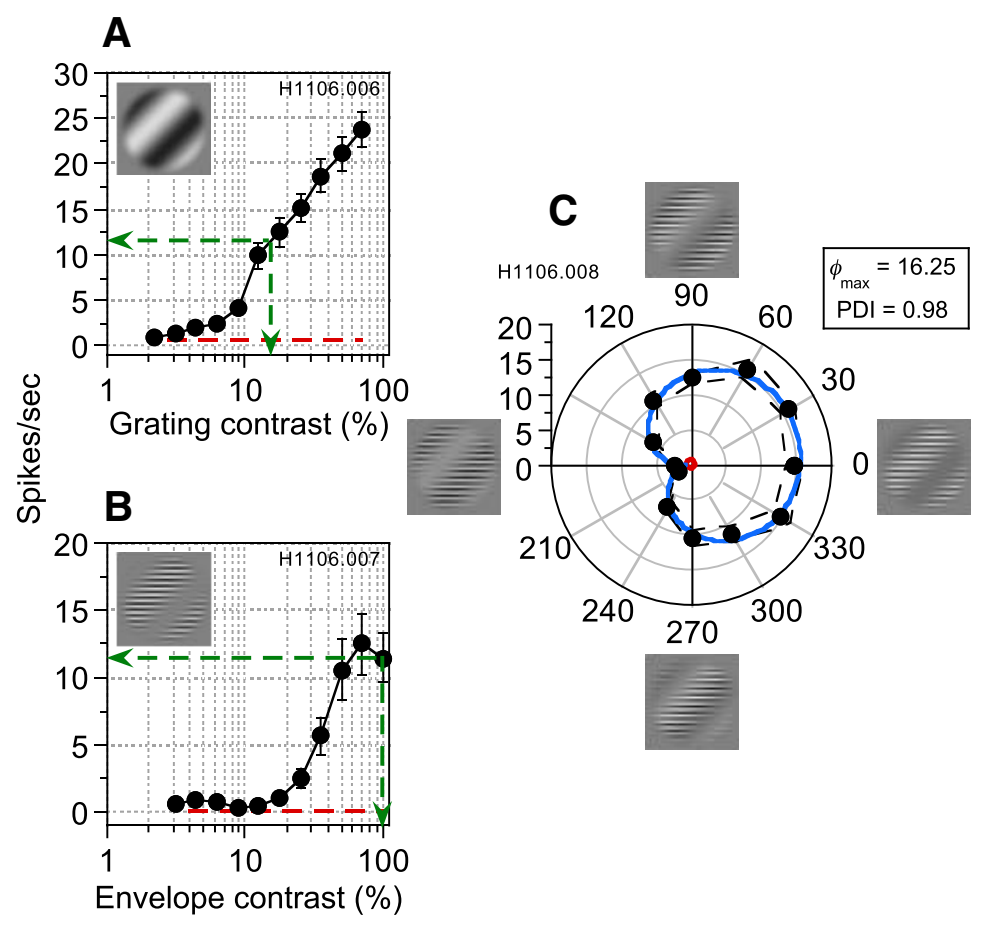

Figure 2. CRFs and phase-dependent interaction for $L M$ and $C M$ stimuli in which parameters are optimized for an example complex-type cell. $\boldsymbol{A}, \boldsymbol{B}$, CRFs for a luminance grating and an envelope, respectively. Error bars indicate $\pm 1 \mathrm{SEM}$. Dashed red lines represent spontaneous activity (responses to a blank field). Dashed green lines show the LM and CM contrast that elicited an equivalent average spike frequency from the neuron. These response-matched contrasts were used to superimpose the grating and envelope at a series of relative phase offsets $\left(0-330^{\circ}\right)$. Both components of the composite stimuli moved together in the neuron's preferred direction. C, Average spike frequencies as a function of relative phase offset of the composite stimuli. Dashed black lines represent \pm 1 SEM. The red line indicates spontaneous activity. This neuron exhibited responses that depended upon the relative phase relationship between LM and CM stimuli, with maximal response when they were superimposed approximately in-phase and minimal response when close to antiphase. These data were well fit (solid blue line) by a descriptive function (Equation 6) used to derive a PDI (Equation 7) and an estimate of the phase offset $\left(\phi_{\max }\right)$ that produced maximal responses.

To quantify the magnitude of spatial phase dependence of a neuron's responses, the measured spontaneous activity was subtracted and the response $R$ as a function of relative spatial phase $\phi$ was fit with a descriptive function as follows:

$$
R=a\left[0.5\left(1+\cos \left(\phi-\phi_{\max }\right)\right)\right]^{0.5}+R_{\min }
$$

where $\phi$ is relative spatial phase between the stimuli, $R_{\min }$ is the minimum response (spikes/s), $a$ is a scaling factor, and $\phi_{\max }$ is the spatial phase producing maximum response $\left(R_{\max }=R_{\min }+a\right)$. This function corresponds to linear vector summation between two sinusoids of equivalent amplitude. $R_{\max }$ would only equal $R_{\min }$ if there were no vector summation (i.e., if the summation process was phase invariant). An example of such a curve fit is shown by the blue contour in Figure $2 C$; for illustration purposes, the spontaneous rate has been added back onto the fitted function values to compare to the data points on the plots that also include the spontaneous rate.

To assess the degree of anisotropy in a neuron's response vs the relative spatial phase, a phase-dependency index (PDI) was calculated as follows:

$$
\mathrm{PDI}=\left(R_{\max }-R_{\min }\right) /\left(R_{\max }+R_{\min }\right)
$$

where $R_{\max }$ and $R_{\min }$ are the maximal and minimal spontaneoussubtracted responses, respectively. This PDI value lies between zero, indicating no phase-dependent interaction (i.e., spike frequency remained relatively constant regardless of the relative spatial phase between LM and CM), and unity, indicating a pronounced interaction (highest degree of anisotropy with a well defined "null" phase having zero response).

Six additional examples of such relative phase responses are shown in Figure 3. In the majority of cases exhibiting a marked phase interaction, maximal responses corresponded to a spatial phase offset close to $0^{\circ}$ (in-phase). However, some neurons responded maximally at other relative spatial phase offsets (for an example, see Fig. 3E). Minimal responses typically occurred at $\sim 180^{\circ}$ relative to the phase offset that produced the maximal response and corresponded to either a distinct null or to a general "flattening" of responses at a number of phase offsets around antiphase. However, the responses of some neurons showed little or no phase dependency (e.g., the complex cell in Fig. $3 D$ ) and were largely invariant regardless of the phase relationship between the two superimposed visual stimuli.

For cells with low PDI, it is possible that the estimated $\phi_{\max }$ could depend heavily on the initial value chosen for the curve fitting procedure. To address this concern, we reran the curve fitting for every neuron using a series of initial $\phi_{\max }$ values. For this, we used a least-squares simplex (Nelder-Mead) method to fit Equation 6 repeatedly to each neuron's spontaneous-subtracted data and varied the initial $\phi_{\max }$ estimate systematically from 0 to $360^{\circ}$ in steps of $1^{\circ}$. The initial estimates for the other curve fit parameters were jittered by $\pm 50 \%$ on each pass. We then found the set of best-fitting parameters that gave the highest goodness-of-fit $\left(R^{2}\right)$ overall for each cell. Therefore, we are confident that the tendency for a $\phi_{\max }$ close to $0^{\circ}$ is not an artifact of initial conditions in the curve-fitting procedure.

A scatterplot of PDI values and $\phi_{\max }$ (degrees) for each neuron in our sample $(N=28)$ is shown in Figure 4. Different neurons displayed a wide range of responses to the combined LM and CM patterns, with many examples exhibiting a "peak" with maximal response at one particular spatial phase and therefore having a PDI substantially greater than zero. A paired-samples $t$ test confirmed that maximal and minimal responses were significantly different $(t=5.829 ; \mathrm{df}=27 ; p<0.0001)$ across the sample population, demonstrating the existence of phase-dependent interactions between LM and CM responses. Regardless of their PDI value, neurons typically produced their maximal responses at spatial phase offsets $\left(\phi_{\max }\right)$ close to $0^{\circ}$. This was true of both simple (circles) and complex cells (triangles) (Fig. 4). Indeed, $86 \%$ of neurons exhibited their peak response at spatial phases within $\pm 45^{\circ}$ of zero. A complete null (PDI $=1.0$ ) was exhibited by $36 \%$ of the neurons. The relationship between PDI and goodness-of-fit $\left(R^{2}\right)$ values derived from fitting Equation 6 is shown in Figure 5A. Although, in principle, a relatively low $R^{2}$ could equally reflect either a weak phase dependency or a jagged (noisy) but strong phase dependence, there is a clear systematic 
trend for low $R^{2}$ values to be associated with the low PDI values, suggesting that it is predominantly a characteristic of cells exhibiting little or no phase selectivity.

Because the anesthesia changed between earlier and later experiments, we investigated whether the anesthesia type was predictive of the degree of phase sensitivity. For each anesthesia type, the PDIs were distributed across the possible range. An independent-samples $t$ test showed that the PDIs did not differ significantly with the type of anesthesia $(t=1.76$; $\mathrm{df}=$ 26 ; $p=0.0902)$. Therefore, we do not believe the change in anesthesia had an effect on the degree of phase sensitivity.

The preference of most neurons for a near-zero phase might suggest that this is a consequence of visual neurons responding better to "dark" than to "light" stimuli (e.g., Jin et al., 2008; Yeh et al., 2009) because there is a perceptual appearance that the dark bars of LM appear more prominent for the in-phase condition (Fig. $1 C, G$ ). However, in our stimuli, the LM was simply linearly added to the CM so that both the light and dark bars/bands of the LM were always physically present (i.e., at all relative spatial phases). From the 1D profiles in Figure 1, $D$ and $H$, it is clear that the net excursions above and below the mean are equivalent for both the in-phase and antiphase stimuli.

Our electrode penetrations were slightly oblique to the surface, traversing all of the laminae down to white matter. However, there was no systematic significant relationship between the PDI value and depth of the recording (Pearson product-moment correlation $r=-0.0248 ; \mathrm{df}=26 ; p=0.9023)$. The neurons with the highest PDI values (1.0) spanned the full range of recorded depths. Therefore, it is highly unlikely that the high PDI cells were concentrated preferentially within a particular range of depths.

To quantify how a given neuron's summation of the two kinds of stimuli differs from simple linear additivity and how this nonlinearity differs from one neuron to another, we also calculated the following ratios:

$$
\begin{aligned}
& \text { Enhancement ratio }=R_{\text {max }} /\left(R_{\text {eq }}-R_{\text {spon }}\right) \\
& \text { Suppression ratio }=R_{\min } /\left(R_{\text {eq }}-R_{\text {spon }}\right)
\end{aligned}
$$

where $R_{\mathrm{eq}}$ is the firing rate of the neuron that was chosen to equate the grating and envelope contrasts of the stimuli used to investigate phase interactions and $R_{\text {spon }}$ is the neuron's spontaneous firing rate. Note that $R_{\text {spon }}$ is not removed in the numerators of these ratios because $R_{\max }$ and $R_{\min }$ are obtained from curve fits to spontaneous-subtracted responses. $R_{\text {eq }}$, however, is a measured response value that includes the spontaneous rate. The $R_{\text {spon }}$ values were measured from the average responses to the blank conditions that were interleaved with the phase conditions in the $\mathrm{LM}+\mathrm{CM}$ experiment. These spontaneous rate values were not significantly different from those similarly obtained from the
LM and CM contrast response measurements, as confirmed with a 1-way, repeated-measures ANOVA $\left(F_{(2,50)}=1.335 ; p=\right.$ $0.2724)$.

One neuron was excluded from this analysis because its $R_{\text {spon }}$ value marginally exceeded its $R_{\mathrm{eq}}$ values. An enhancement ratio of two (red dashed line in Fig. $5 B$ ) indicates that the maximal response $\left(R_{\max }\right)$ of the cell is exactly twice as much to both stimuli together as to each in isolation (linear summation). Similarly, a suppression ratio of zero (blue dashed line in Fig. $5 B$ ) indicates complete nulling of the neuron's response when the stimuli are in antiphase $\left(R_{\min }\right)$ relative to $\phi_{\max }$. Enhancement ratios spanned 0.627 to 4.209 (mean $=2.082)$ and suppression ratios spanned 1.647 to -0.933 (mean $=0.342)$, indicating considerable heterogeneity among our neuron population (Fig. $5 B$ ). There was a moderate tendency for the magnitude of the suppression ratio to decrease as PDI increased, indicating a greater suppressive influence for neurons that exhibited the largest phase dependencies. Whether neurons were of the simple or complex type did not affect either ratio systematically.

To confirm the appropriateness of our LM and CM responsematching procedure, for a number of neurons, we measured phase-dependent interactions between LM and CM at two different response-matched contrasts. An example from a simple-type neuron is shown in Figure 6. LM (Fig. 6A) and CM (Fig. 6B) contrasts were matched at either 14 (purple dotted lines) or 28 

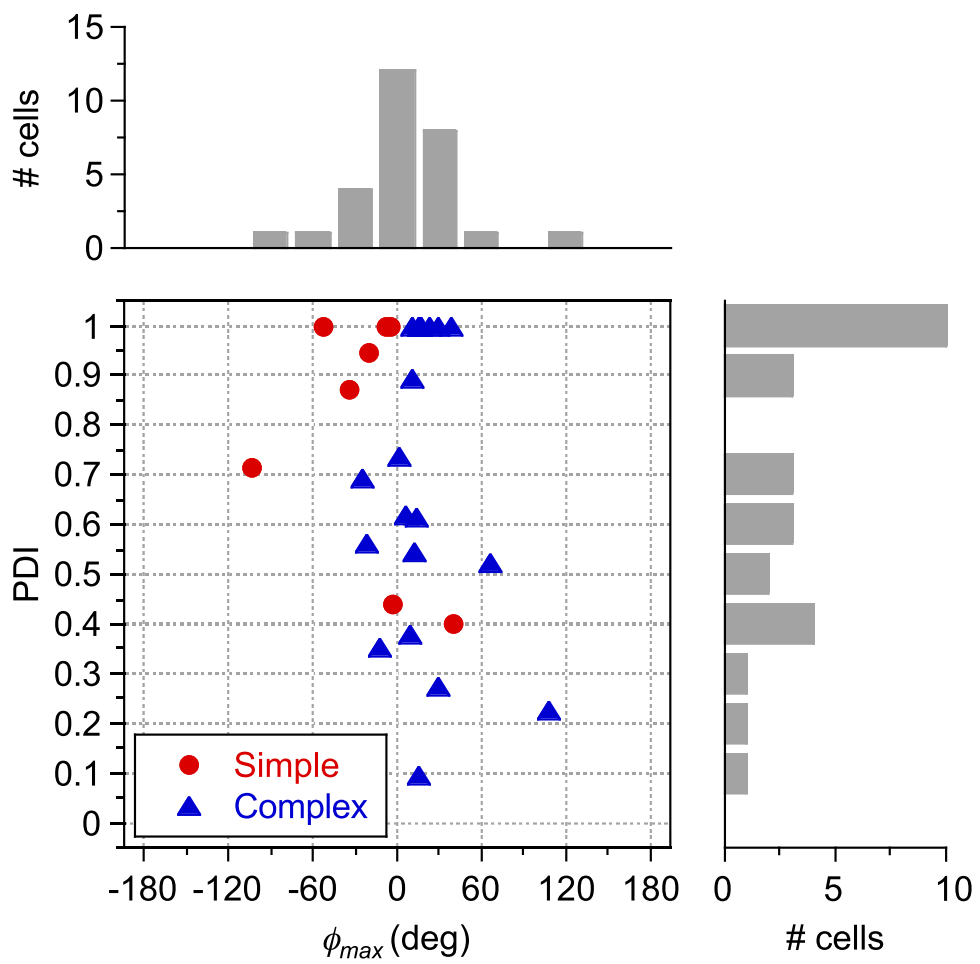

Figure 4. PDIs plotted against optimal phase alignments $\left(\phi_{\max }\right)$ for all neurons in the sample $(N=28)$. Simple-type neurons are denoted by red circles and complex-type by blue triangles. Marginal histograms show the distribution of $\phi_{\max }$ (top) and PDI (right) values within the sample population. $\phi_{\max }$ ranged from -103.42 to 107.35 , with a mean of $6.57^{\circ}$. PDI values ranged from 0.09 to 1.0 , with a mean of 0.71 , indicating a wide range of relative phase dependencies in different neurons.

A

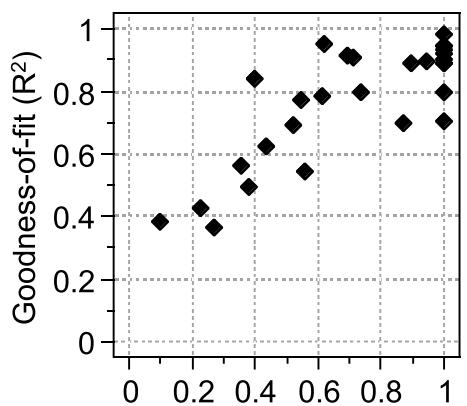

B

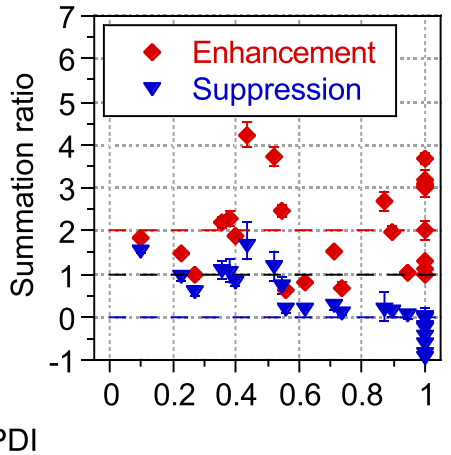

PDI

Figure 5. Goodness-of-fit and summation ratios for phase-dependent interactions. $A, R^{2}$ values derived from fitting Equation 6 to each neuron's responses to the combined LM and CM patterns plotted against the corresponding PDI values. Equation 6 best fit responses of neurons that exhibited a high degree of interaction with a well defined null phase (PDI values $\sim$ unity). $\boldsymbol{B}$, Enhancement and suppression ratios (Equations 8 and 9, respectively) indicating neurons' responses to LM and CM stimuli in isolation compared with responses to their composite at $\phi_{\max }$ (enhancement ratio, red diamonds) and $\phi_{\max }-180^{\circ}$ (suppression ratio, blue triangles). An enhancement ratio of two (red dashed line) indicates that the $R_{\max }$ of the cell is exactly twice as much as both stimuli together as to each in isolation (linear summation). A suppression ratio of zero (blue dashed line) indicates complete nulling of the neuron's response at $R_{\min }$. Different neurons exhibited a range of enhancement and suppression ratios not always consistent with simple linear summation. Error bars around each of these ratios indicate $68 \%$ confidence intervals ( equivalent to $\pm 1 \mathrm{SE}$ ) generated by a nonparametric, bias corrected and accelerated (BCa) bootstrapping technique that created 10,000 bootstrapped replications of each fitted function without assuming a Gaussian distribution for the raw data or the residuals (Efron and Tibshirani, 1993).

(green dotted lines) spikes/s. Comparable phase dependence was evident at both response-matched amplitudes (14 spikes/s; Fig. $6 C ; 28$ spikes/s; Fig. 6D), with similar $\phi_{\max }$ and PDI values for each, thereby verifying the robustness of our matching paradigm and confirming that the absolute firing rate chosen to equate the two types of stimuli was not critical to the pattern of results found.

Some of the sampled neurons were simple-type cells and thus had modulated responses to the drifting LM or CM stimuli. We wondered whether analysis of the temporal phases of these responses might be related to the dependence on relative spatial phase of LM and CM stimuli. To investigate this, we examined the temporal phase of the first harmonic at the equated contrast value in the contrast response measurements (interpolating where necessary) for LM and CM gratings. Figure $7 A$ shows that the amount of phase interaction, PDI, did not show a significant relationship with the difference in temporal phases for LM and CM responses (Pearson product moment correlation coefficient $r=-0.4750 ; \mathrm{df}=6 ; p=$ 0.2342 ), although this may not be surprising in view of the small sample size. However, in Figure $7 B, \phi_{\max }$ shows a clear and statistically significant positive association $(r=0.9088 ; \mathrm{df}=6 ; p=0.0018)$ with the temporal phase difference. As the temporal phase difference increases, the $\phi_{\text {max }}$ also increases systematically. Therefore, it looks like a lawful and expected relationship, for the simple cells at least, that the variation in $\phi_{\max }$ away from a relative spatial phase of zero is driven by the difference in the temporal phases of the response to the two types of stimulus.

\section{Amplitude-dependent responses}

Neurons typically exhibited an enhanced response when LM and CM stimuli were phase aligned and a diminished response at or around antiphase (Figs. 2C, 3, 6C,D). However, the magnitude of the neuronal response might be determined, not only by the spatial phase offset between LM and CM, but possibly also by other factors such as the relative amplitudes of the two spatially superimposed stimuli. When LM and CM stimuli were equated in terms of response, neurons produced a null or minimum response at antiphase compared with their in-phase response. This is presumably because, in the former condition, LM and CM effectively cancelled each other out (Fig. 1G) and no net driving signal was available to the neuron. At antiphase, effective visual information can be reintroduced by increasing the amplitude of one stimulus relative to the other so that they are no longer effectively balanced. If one stimulus drives the neuron more strongly than the other, then the nulling would be abolished and the neuron should become more responsive. To test this notion, we fixed the amplitude of the CM stimulus at the value used to measure phase-dependent interactions and varied the contrast of the LM 
stimulus at the neuron's null phase so that it was less than, greater than, or equal to that derived from the response-matching procedure (green arrows in Fig. 8). When stimuli were superimposed in antiphase with their amplitudes carefully equated, the neuron produced a minimal response. However, when the LM contrast was either reduced or increased beyond this match point, the neuron's response increased as the two superimposed stimuli became progressively mismatched. Figure $8, B-E$, shows results from a further four representative neurons. The precise nature of the interaction varied according to the contrast range used in each neuron, which was determined by the CRFs for each stimulus type and constrained by the requirement that the sum of the LM grating contrast and CM carrier contrast cannot exceed $100 \%$. Among the examples of these measurements shown in Figure 8, some cells exhibited responses that were reasonably symmetrical around the central match point (Fig. $8 A, B, D$ ), indicating that LM and CM were well equated at this contrast level. In some cases, the responses were appreciably less symmetrical, which may be due in part to imperfect equating of the stimulus components (Fig. $8 C$ ) or the limited contrast range available (Fig. $8 E$ ).

\section{Discussion}

We have shown that neurons in early visual cortex, which respond form-cue invariantly to firstorder LM and second-order CM, responded in a systematic manner to the relative spatial phase offset between the two kinds of patterns when they were superimposed. In both simple- and complex-type cells, maximal responses typically occurred when response-equated LM and CM were superimposed at or close to phase alignment, with a minimal response when in antiphase. In many cases, maximal and minimal responses were markedly different to varying degrees in different neurons. Neurons varied substantially in the relative roles of suppressive or facilitative interaction effects. The degree of this interaction between LM and CM at antiphase could be modified by increasing the amplitude of one stimulus relative to the other. When the LM amplitude was either reduced or increased around a fixed CM amplitude, responses increased as the two superimposed stimuli became progressively mismatched.

An important concern in experiments using CM stimuli is that the observed neuronal responses might be due to "distortion products" from nonlinearities of the display device or the photoreceptors (MacLeod et al., 1992; Zhou and Baker, 1994). Such artifactual responses would occur regardless of carrier pattern characteristics. CM responses here were tuned selectively to relatively high values of carrier spatial frequency well outside of the luminance passband and thus were highly unlikely to be artifactual. The phase dependence of the response to combined LM and CM could arise in a similarly artifactual manner. However, in that case, the optimal phase value would always be the same; for example, an early expansive nonlinearity would always give $\phi_{\max }=0^{\circ}$. This is because an expansive nonlinearity introduces a distortion product into the neural representation of a contrast-modulated image with the same frequency and phase as the modulating waveform (see Fig. 1 of Smith and Ledgeway, 1997) that will combine with a superimposed luminance grating of the same spatial phase to produce a maximal response. We observed a considerable scatter in values of optimal phase in different neurons, again making such a possibility highly unlikely.

It is entirely possible that we may have missed some relevant neurons due to our protocol. Our neuron search stimulus was a bar of light and, as such, would not reveal neurons that were responsive to only CM stimulus attributes or even possibly a CM-driven neuron in which the response to CM can be modulated by LM. We only examined neurons that responded both to LM and to CM in isolation, so we might have missed, for example, neurons that are unresponsive to $\mathrm{CM}$ in isolation, but with an LM response that is affected differentially by superposition of CM stimuli in different relative phases. Moreover, there might exist neurons that respond only to specific stimulus combinations, but not to LM or CM stimuli alone. Currently, there is no evidence for the existence of neurons having such highly nonlinear summation, but if they were present, we would have missed them.

\section{Psychophysical studies of LM and CM mixtures}

Psychophysical studies have examined the degree to which firstand second-order cues interact perceptually when they are spa- 
A

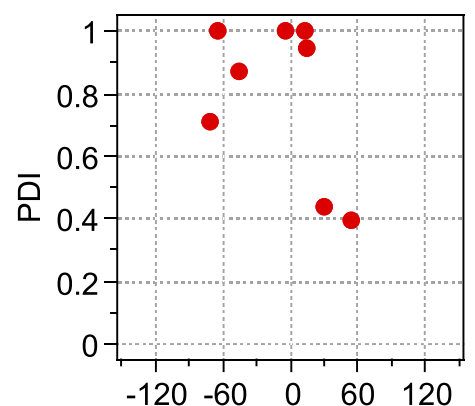

LM - CM phase difference (deg)

Figure 7. Relationship of $L M+C M$ phase interactions to temporal phase lags of responses in simple-type cells. $\boldsymbol{A}$, Amount of $\mathrm{PDI}$ as a function of difference in temporal phase lag measured for LM and for CM stimuli presented alone in simple-type cells having modulated discharges. $\boldsymbol{B}$, Same as $\boldsymbol{A}$, but optimal phase $\left(\phi_{\max }\right)$ for response to $\mathrm{LM}+\mathrm{CM}$ compound stimuli showing an approximately linear relationship.
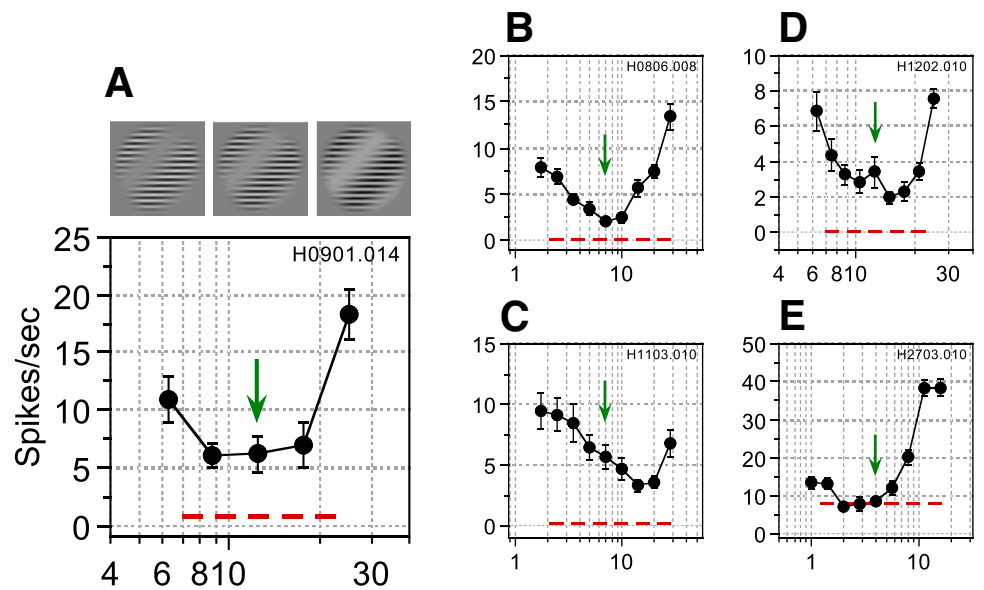

Grating contrast (\%)

phase with the CM and its amplitude was varied in an attempt to null the hypothetical distortion product. They found that direction identification performance was unimpaired by the presence of the moving LM. Lu and Sperling (1995) also found no appreciable phase dependency when performance was measured for combinations of drifting LM and CM noise matched for spatial frequency and effective amplitude, although others (Scott-Samuel and Georgeson, 1999; Allard and Faubert, 2013) have reported phase dependence, but only at high temporal frequencies $(15 \mathrm{~Hz})$. Studies using stationary patterns are also equivocal with regard to the influence of relative spatial phase. Some have found moderate to strong phase selectivity (Henning et al., 1975; Nachmias, 1989), whereas others have reported that masking magnitude is independent of phase (Cropper, 1998; Willis et al., 2000). A complication is that other factors such as extended practice, individual differences, local luminance cues in the image, and the predictability of the phase relationships on each trial are also known to influence performance on this task (Nachmias and Rogowitz, 1983; Badcock, 1984). One possibility that could reconcile these discrepant results is that the human visual system contains neurons responsive to both LM and $\mathrm{CM}$, but with a range of phase selectivity (cf. Fig. 3). Performance in a given situation could depend on which neurons are most sensitive, giving rise to either phase-independent or phase-specific masking.

\section{Neural mechanisms}

In early visual cortex of the cat and the macaque, a substantial fraction of the neurons respond both to first- and

tially superimposed. For example, Smith and Scott-Samuel (1998) showed that spatial frequency discrimination and speed discrimination could be enhanced when first- and second-order gratings were superimposed compared with when each was presented alone. Similarly, Johnson et al. (2007) found that texture discrimination was enhanced or impaired depending on whether the local elements comprising the textures contained spatially correlated or uncorrelated LM and CM information, respectively.

Masking studies have also investigated whether LM and CM gratings interact in a phase-specific manner, the underlying assumption being that, if the two types of stimuli are encoded by a common mechanism, then detection should be highly dependent on the two patterns' relative spatial phase. For example, Badcock and Derrington (1989) explored the possibility that second-order motion, defined by variations in contrast, is detected on the basis of a distortion product, by adding a moving sine grating (LM) to a drifting beat (CM) pattern of the same spatial frequency. The LM was $180^{\circ}$ out of second-order patterns (Zhou and Baker, 1994; Li et al., 2014). Most proposed models of such responses involve two parallel signal processing pathways, each specialized for one or the other type of stimulus, the signals of which are then combined (Mareschal and Baker, 1999). Alternatively, cortical second-order responses could originate from LGN (and ultimately retinal) Y-cells, the responses of which carry both luminance information at low spatial frequencies and specificity for carrier attributes at high frequencies (Rosenberg and Issa, 2011). The present findings of phase-dependent combination are not incompatible with either of these schemes. Models based on human psychophysics have involved separate early detection of the two kinds of stimuli, with subsequent interactions at a later stage (Georgeson and Schofield, 2002). A model with crosswise gain control interactions between pathways carrying a mixture of first-and second-order information (Schofield et al., 2010; Sun and Schofield, 2011) predicts our observations of stronger responses to in-phase than antiphase conditions. 
As a baseline reference, it is worth considering that a cortical neuron might just linearly add the separately computed responses to LM and CM stimuli. In the case of a simple-type cell, the modulated responses to the LM and CM stimuli would sum maximally at one phase and cancel out at the opposite phase, giving a PDI approaching unity. In fact, the optimal relative phase values were linearly predictable from the phase lags of the LM and CM alone (Fig. $7 B$ ). The lack of relationship to the PDI value (Fig. $7 A$ ) may be because the effect of the temporal phase lag is to effectively shift the $\phi_{\max }$ value in a neuron that already is or is not phase selective. Complex-type cells might be thought of as linearly adding energy-like responses to LM and CM stimuli, which would not be modulated and thus their summation should be phase invariant $(\mathrm{PDI}=\sim 0$ ). Alternatively, a complex cell might result from an energy-type operation on pooled responses of simple cell (modulated) responses to LM and CM stimuli, early summation of which would give a high PDI. In our sample, the complex-type cells showed a wide range of PDI values (Fig. 4), suggesting a continuum between such types of models.

\section{Functional implications/significance}

These neurons show complex interactions between both amplitude and phase of LM and CM components, which are in some cases consistent with vector summation. This finding suggests a modification of the form-cue invariance principle (Albright, 1992). Although these neurons are form-cue invariant to orientation, spatial frequency, and motion direction, they are in most cases not invariant to the relative phase of superimposed first- and second-order components.

These properties might have implications for how the visual system processes natural images. Neurons with little or no $\mathrm{LM}+\mathrm{CM}$ phase dependence would respond to boundaries regardless of the configuration of their components, whereas those having a strong phase dependency would respond selectively to particular co-occurrences of first- and second-order information in natural images (Johnson and Baker, 2004). These neurons' responses carry information that may help to disambiguate whether luminance changes in the retinal image arise from surface reflectance changes or from illumination gradients such as shading or shadows (Schofield et al., 2006, 2010; Sun and Schofield, 2011). More generally, the heterogeneity in degree of phase-dependent interactions and suppression versus enhancement might provide a basis for disambiguating or decoding a variety of different kinds of boundaries. A promising future direction would be to examine the relative phases of LM and CM components at boundaries in natural images that arise from different causes.

\section{References}

Albright TD (1992) Form-cue invariant motion processing in primate visual cortex. Science 255:1141-1143. CrossRef Medline

Allard R, Faubert J (2013) No second-order motion system sensitive to high temporal frequencies. J Vis 13: pii: 4. CrossRef Medline

Badcock DR (1984) Spatial phase or luminance profile discrimination. Vision Res 24:613-623. CrossRef Medline

Badcock DR, Derrington AM (1989) Detecting the displacement of spatial beats: no role for distortion products. Vision Res 29:731-739. CrossRef Medline

Brainard DH (1997) The psychophysics toolbox. Spat Vis 10:433-436. CrossRef Medline

Chaudhuri A, Albright TD (1997) Neuronal responses to edges defined by luminance vs temporal texture in macaque area V1. Vis Neurosci 14:949962. CrossRef Medline
Cropper SJ (1998) Detection of chromatic and luminance contrast modulation by the visual system. J Opt Soc Am A Opt Image Sci Vis 15:19691986. CrossRef Medline

Efron B, Tibshirani RJ (1993) An introduction to the bootstrap. London: Chapman and Hall.

El-Shamayleh Y, Movshon JA (2011) Neuronal responses to texturedefined form in macaque visual area V2. J Neurosci 31:8543-8555. CrossRef Medline

Geesaman BJ, Anderson RA (1996) The analysis of complex motion patterns by form/cue invariant MSTd neurons. J Neurophysiol 16:47164732. Medline

Georgeson MA, Schofield AJ (2002) Shading and texture: Separate information channels with a common adaptation mechanism? Spat Vis 16:59-76. CrossRef Medline

Hallum LE, Landy MS, Heeger DJ (2011) Human primary visual cortex is selective for second-order spatial frequency. J Neurophysiol 105:21212131. CrossRef Medline

Henning GB, Hertz BG, Broadbent DE (1975) Some experiments bearing on the hypothesis that the visual system analyzes patterns in independent bands of spatial frequency. Vision Res 15:887-897. CrossRef Medline

Jin JZ, Weng C, Yeh CI, Gordon JA, Ruthazer ES, Stryker MP, Swadlow HA, Alonso JM (2008) On and off domains of geniculate afferents in cat primary visual cortex. Nat Neurosci 11:88-94. CrossRef Medline

Johnson AP, Baker CL Jr (2004) First- and second-order information in natural images: a filter-based approach to image statistics. J Opt Soc Am A Opt Image Sci Vis 21:913-925. CrossRef Medline

Johnson AP, Prins N, Kingdom FA, Baker CL Jr (2007) Ecologically valid combinations of first- and second-order surface markings facilitate texture discrimination. Vision Res 47:2281-2290. CrossRef Medline

Kleiner M, Brainard D, Pelli D (2007) What's new in Psychtoolbox-3? Perception 36:1.

Larsson J, Landy MS, Heeger DJ (2006) Orientation-selective adaptation to first- and second-order patterns in human visual cortex. J Neurophysiol 95:862-881. Medline

Ledgeway T, Zhan C, Johnson AP, Song Y, Baker CL Jr (2005) The direction selective contrast response of area 18 neurons is different for first- and second-order motion. Vis Neurosci 22:87-99. CrossRef Medline

Li G, Yao Z, Wang Z, Yuan N, Talebi V, Tan J, Wang Y, Zhou Y, Baker CL Jr (2014) Form-cue invariant second-order neuronal responses to contrast modulation in primate area V2. J Neurosci 34:12081-12092. CrossRef Medline

Lu ZL, Sperling G (1995) The functional architecture of human visual motion perception. Vision Res 35:2697-2722. CrossRef Medline

MacLeod DI, Williams DR, Makous W (1992) A visual nonlinearity fed by single cones. Vision Res 32:347-363. CrossRef Medline

Mareschal I, Baker CL Jr (1999) Cortical processing of second-order motion. Vis Neurosci 16:527-540. Medline

Nachmias J (1989) Contrast modulated maskers: test of a late nonlinearity hypothesis. Vision Res 29:137-142. CrossRef Medline

Nachmias J, Rogowitz BE (1983) Masking by spatially modulated gratings. Vision Res 23:1621-1629. CrossRef Medline

Nishida S, Sasaki Y, Murakami I, Watanabe T, Tootell RB (2003) Neuroimaging of direction-selective mechanisms for second-order motion. J Neurophysiol 90:3242-3254. CrossRef Medline

Pelli DG (1997) The VideoToolbox software for visual psychophysics: transforming numbers into movies. Spat Vis 10:437-442. CrossRef Medline

Rosenberg A, Issa NP (2011) The Y cell visual pathway implements a demodulating nonlinearity. Neuron 71:348-361. CrossRef Medline

Schofield AJ (2000) What does second-order vision see in an image? Perception 29:1071-1086. CrossRef Medline

Schofield AJ, Hesse G, Rock PB, Georgeson MA (2006) Local luminance amplitude modulates the interpretation of shape-from-shading in textured surfaces. Vision Res 46:3462-3482. CrossRef Medline

Schofield AJ, Rock PB, Sun P, Jiang X, Georgeson MA (2010) What is second-order vision for? Discriminating illumination versus material changes. J Vis 10:2. CrossRef Medline

Scott-Samuel NE, Georgeson MA (1999) Does early non-linearity account for second-order motion? Vision Res 39:2853-2865. CrossRef Medline

Seiffert AE, Somers DC, Dale AM, Tootell RB (2003) Functional MRI studies of human visual motion perception: texture, luminance, attention and after-effects. Cereb Cortex 13:340-349. CrossRef Medline 
Skottun BC, De Valois RL, Grosof DH, Movshon JA, Albrecht DG, Bonds AB (1991) Classifying simple and complex cells on the basis of response modulation. Vision Res 31:1079-1086. Medline

Smith AT, Ledgeway T (1997) Separate detection of moving luminance and contrast modulations: fact or artifact. Vision Res 37:45-62. CrossRef Medline

Smith AT, Scott-Samuel NE (1998) Stereoscopic and contrast-defined motion in human vision. Proc Biol Sci 265:1573-1581. CrossRef Medline

Sun P, Schofield AJ (2011) The efficacy of local luminance amplitude in disambiguating the origin of luminance signals depends on carrier frequency: Further evidence for the active role of second-order vision in layer decomposition. Vision Res 51:496-507. CrossRef Medline
Tanaka H, Ohzawa I (2006) Neural basis for stereopsis from second-order contrast cues. J Neurosci 26:4370-4382. CrossRef Medline

Tusa RJ, Rosenquist AC, Palmer LA (1979) Retinotopic organization of area 18 and 19 in the cat. J Comp Neurol 185:657-678. CrossRef Medline

Willis A, Smallman HS, Harris JM (2000) Comparing contrast-modulated and luminance-modulated masking: effects of spatial frequency and phase. Perception 29:81-100. CrossRef Medline

Yeh CI, Xing D, Shapley RM (2009) "Black" responses dominate macaque primary visual cortex V1. J Neurosci 29:11753-11760. CrossRef Medline

Zhou YX, Baker CL Jr (1994) Envelope-responsive neurons in area 17 and 18 of cat. J Neurophysiol 72:2134-2150. Medline 